\title{
Micromechanical formulation of the mobilized stress ratio under principal stress rotation in granular materials
}

\author{
Homayoun Shaverdi ${ }^{1}$, Farzin Kalantary ${ }^{2}$, Mohd R. Taha ${ }^{3}$ \\ ${ }^{1}$ Civil Engineering Department, Ilam University, Iran \\ ${ }^{2}$ Civil Engineering Department, K.N. Toosi University of Technology, Iran \\ ${ }^{3}$ Civil and Structural Engineering Department, Universiti Kebangsaan Malaysia(UKM), Malaysia
}

\begin{abstract}
In this paper the mobilized stress ratio is investigated by using the static equilibrium at the micro scale level analysis. The mobilized stress ratio is obtained in the distribution of the contact normal, $E(\beta)$, and the inter-particle mobilized friction angle, $\phi_{\mu}$. The second invariant of the fabric tensor, $\alpha$ is used to account for fabric and its evolution. There is a difference between the theoretical rotation of principal stress axes that is obtained by the classical equations of the mechanics of materials and the experimental rotation of principal stress axes. The experimental rotation is related to the anisotropic parameter, $\alpha$. The noncoincidence between the theoretical and experimental is related to the relative direction of the mobilized plane and the bedding plane. A comparison with experimental tests demonstrates the validity of this formulation.
\end{abstract}

\section{Introduction}

Experimental tests have shown that the gradual rotation of the principal stress axes during the progressive shearing of granular materials cause the gradual rotation of the preferred direction of the contact normal (Oda \& Konishi, 1974, Oda, 1975, Arthur et al., 1977, Miura et al., 1986, Yang et al., 2016). Roscoe et al., (1967) showed that the rotation of the principal stress axes happens during simple shear of sand. They found that the rotation of the principal axes occurs when the shear stress $\tau$ increases and they also found that the inclination angle, $\psi$, of the maximum principal stress axis with respect to the vertical direction tends to approach a certain asymptotic value. There is the noncoincidence between the principal stress direction and the principal strain rate direction when the applied shear loads are rotating (Arthur et al., 1977, Wong \& Arthur, 1986, Gutierrez et al., 1991, Yang et al., 2016). At the start of shearing there is a non-coaxiality between stress and fabric, and also between strain rate and fabric. Shaverdi et al. (2014) showed that fabric has a bridgelike role in the shear deformation. The contact normal tends to be coaxial with the principal stress direction during shear, while the principal strain increment also tends to be coaxial but at a different rate. At large shear strain, stress, strain and fabric will coincide. The contact normal are the linkage between stress and strain.

Oda (1972) and Oda \& Konishi (1974) observed that the intensity of the preferred orientation of the contact normal (fabric anisotropy). In other words, fabric anisotropy has a major effect on the shear strength of granular materials in the presence of the rotation of the principal stress axes (Roscoe et al., 1970, Oda, 1972, Cai et al., 2013, Yang et al., 2016). The stress and strain are linked via the contact normal. The major direction of fabric anisotropy always lays between stress and strain principal directions. This non-coaxiality between stress and strain rate directions result in the difference between the real direction of the major principal stress and the theoretical direction. By micromechanical analysis Oda \& Konishi (1974) and Yang et al. (2016) among others showed that the direction of the contact forces are different from the direction of the contact normal. Many researchers have also shown that the rotation of principal stress directions have to be related to the fabric anisotropy parameters. Oda \& Konishi (1974) by considering the static equilibrium at the micro scale analysis proposed the following equation:

$$
\begin{aligned}
& \left\{J_{1} \tan ^{2}\left(45+\frac{\phi_{\mu}}{2}\right)-J_{2}\right\} \tan ^{2} \psi-\left(J_{3}-1\right)\left\{\tan ^{2}\left(45+\frac{\phi_{\mu}}{2}\right)-1\right\} \tan \psi \\
& +\left\{J_{1}-J_{3} \tan ^{2}\left(45+\frac{\phi_{\mu}}{2}\right)\right\}=0
\end{aligned}
$$

where: $J_{1}=\frac{\int_{-\pi / 2}^{\pi / 2} 2 E(\beta) \sin \beta \mathrm{d} \beta}{\int_{0}^{\pi} 2 E(\beta) \sin \beta \mathrm{d} \beta}$

\footnotetext{
* Corresponding author: h.shaverdi@ilam.ac.ir, homayoun_shaverdi@yahoo.com, ${ }^{2}$ fz kalantary@,kntu.ac.ir, ${ }^{3}$ profraihan@gmail.com
} 
$J_{2}=\frac{\int_{0}^{\pi} 2 E(\beta) \cos \beta \mathrm{d} \beta}{\int_{0}^{\pi} 2 E(\beta) \sin \beta \mathrm{d} \beta}, J_{3}=\frac{\int_{-\pi / 2}^{\pi / 2} 2 E(\beta) \cos \beta \mathrm{d} \beta}{\int_{0}^{\pi} 2 E(\beta) \sin \beta \mathrm{d} \beta}$

where $E(\beta)$ is the distribution of the contact normal, $\beta$ is the direction of the contact normal with respect to the principal stress direction, $\psi$ is the direction of the principal stress axes with the vertical direction. This equation cannot easily be applied to determine the direction of the major principal stress. In order to obtain and develop a practicable equation to calculate the direction of the major principal stress, the micro-level parameters that have been developed by Shaverdi \& Taha (2013) and Shaverdi et al., (2014) are used. These parameters are related to the macro-level variables such as the shear stress, the confining pressure and the mobilized stress ratio.

\section{Theoretical considerations}

Rothenburg \& Bathurst (1989) showed that the distribution of the contact normal can be represented by the following equation:

$$
E(\beta)=\frac{1}{2 \pi}\left(1+\alpha \cos 2\left(\beta-\beta_{f}\right)\right)
$$

where $\alpha$ is the degree of anisotropy and $\beta_{f}$ is the direction of the major principal fabric.

Putting Eq.(3) into Eq.(2) and some manipulation we will find:

$$
J_{1}=J_{2}=\frac{2 \alpha \sin 2 \beta_{f}}{3-\alpha \cos 2 \beta_{f}}, J_{3}=\frac{3+\alpha \cos 2 \beta_{f}}{3-\alpha \cos 2 \beta_{f}}
$$

Incorporating Eq.(4) into Eq.(1) and solve the second order equation to obtain $\tan \psi$, the essential equation to calculate the rotation of principal stress will be obtain as follows:

$$
\tan \theta_{\sigma r}=\tan \psi=\frac{\left(1-J_{3}\right) \pm \sqrt{\left(J_{3}-1\right)^{2}+4 J_{1}}}{2 J_{1}}
$$

It is obvious that the real rotation of the principal stress is dependent on the contact normal distribution functions (fabric anisotropy). Shaverdi et al. (2014) and Taha \& Shaverdi (2013) proposed some equations to calculate the degree of anisotropy $\alpha$ and the direction of the fabric tensor during shear process, $\beta_{f}$. As mentioned already, the rotation of the principal stress axes have to be related to the fabric anisotropy parameters only. Eq. (5) can micromechanically predict the variation of the principal stress axes by considering the fabric anisotropy parameters $\left(\alpha, \beta_{f}\right)$. The required equation is developed to consider the direction of the major principal stress direction by micro-level parameters. In the shearing process, these parameters $\left(\alpha, \beta_{f}\right)$ can be obtained by macro-level quantities such as the shear stress and the confining pressure. Unlike the Oda \& Konishi's (1974) equation, that required the distribution of the contact normals $\left(J_{1}, J_{2}, J_{3}\right)$ and the internal friction angle $\left(\phi_{\mu}\right)$, the developed equation can calculate the direction of the major principal stress by using the macro quantities directly (because $\alpha$ and $\beta_{f}$ can be obtained from the ratio of the shear to normal stresses and the variation of the shear stress).

\section{Mobilized stress ratio in simple shear test}

Contact force transmitted through the contact surface from the grain $P_{i}$ to the grain $P_{i}^{\prime}$ can be resolved in the principal directions $X, Y$. As shown in Fig.1, the interparticle sliding have to occur when the following equation is satisfied:

$$
\frac{F_{y^{\prime} i}}{F_{x^{\prime} i}}=\tan \left(\frac{\pi}{2}+\phi_{\mu}-\beta^{\prime}\right)
$$

Considering the distribution low of contact forces by Oda (1972) and Horne (1956), the contact forces can be resolved in the principal stress axes $X^{\prime}$ and $Y^{\prime}$. Finally, we will obtain (due to the limitation of space we cannot explanation other steps)(Oda \& Konishi, 1974):

$$
\frac{\tau}{\sigma}=\frac{\left\{1+\tan ^{2}\left(45+\frac{\phi_{\mu}}{2}\right)\right\} K+\left\{\tan ^{2}\left(45+\frac{\phi_{\mu}}{2}\right)-1\right\} \tan \psi}{\tan ^{2} \psi+\tan ^{2}\left(45+\frac{\phi_{\mu}}{2}\right)+\left\{\tan ^{2}\left(45+\frac{\phi_{\mu}}{2}\right)-1\right\} K \tan \psi}
$$

where: $\quad K=\frac{4}{3} \frac{\alpha \sin 2 \beta_{f}}{\left(-2+\alpha \cos 2 \beta_{f}\right)}$

Eqs.(7) and (8), show that the mobilized stress ratio is related to the fabric anisotropy $\left(\alpha, \beta_{f}\right)$ and the interparticle friction angle $\left(\phi_{\mu}\right)$. The anisotropic parameters and their evolution can be obtained by the relationships that have been developed by Shaverdi et al. (2014). The theoretical mobilized stress ratio can be compared with the experimental mobilized stress ratio (Eqs. (7) and (8)), as shown in Fig.2.

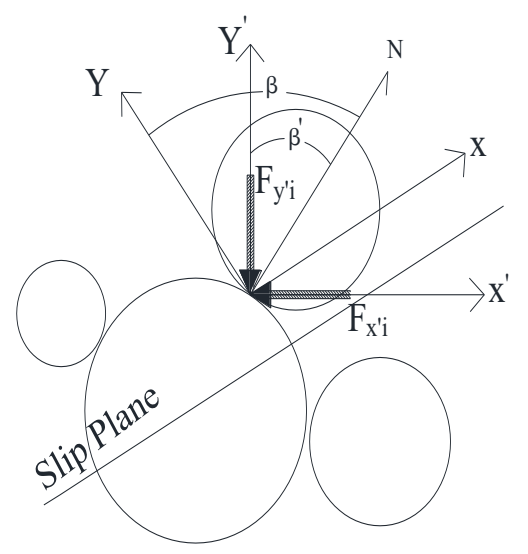

Fig. 1. Contac forces resolved in principal stress axes $X^{\prime}$ and $Y^{\prime}$ 


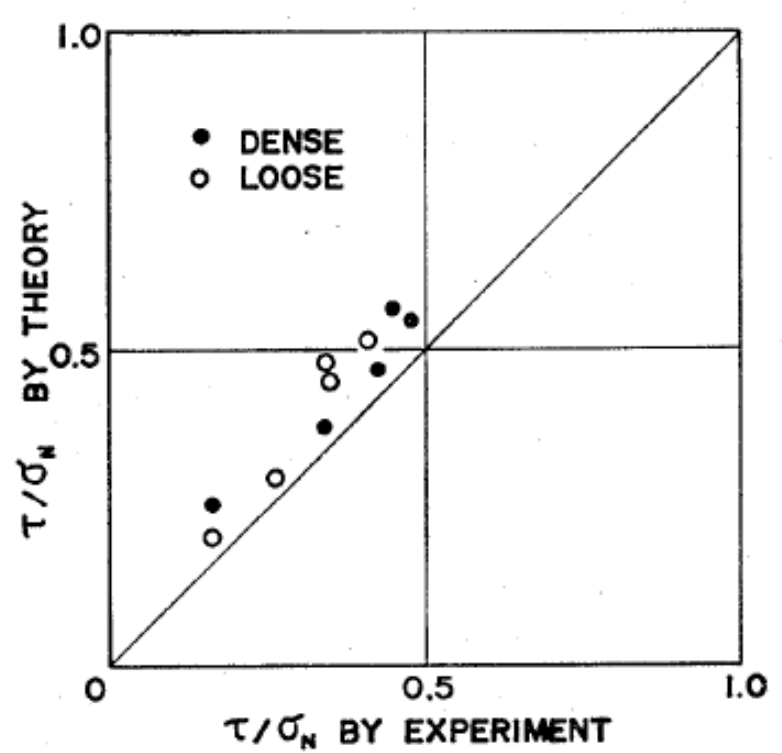

Fig. 2. Relationship between experimentally determined $\tau / \sigma_{N}$ values and calculated ones of two dimensional granular model deformed under simple shear (after Oda \& Konishi 1974)

\section{Verification with the experimental tests}

The theoretical rotation of the principal stress and the direction of the major principal stress can be obtained as follows:

$$
\tan 2 \theta_{\sigma t}=\frac{2 \tau_{x y}}{\sigma_{x}-\sigma_{y}}
$$

The proposed equation (Eq.(5)) can be compared with the theoretical equation (Eq.(9)), as shown in Fig.3. It can be seen that this equation can well predict the rotation of principal stress.

Oda \& Konishi (1974) conducted some experimental tests by using photo elastic materials. They were obtained the real direction of the major principal stress. By the methods developed by Budhu (1985) and Ochiai (1975) to calculate the theoretical rotation of the principal stress axes (Eq.(9)) we found that there is a difference between the real direction of the major principal stress and the theoretical of it. The actual direction of the major principal stress is obtained from the equation proposed by the authors that corresponds exactly to the direction proposed by Oda \& Konishi (1974). In the other words, the comparisons Figs. 3a \& $3 \mathrm{~b}$, the actual direction of principal stress is obtained from Eq. (5) and the theoretical direction is obtained from Eq. (9).As proposed by Oda \& Konish (1974), the interparticle friction angle for the dense specimens (D) is $\phi_{\mu}=26^{\circ}$ and for the loose ones (L) is $\phi_{\mu}=22^{\circ}$. the difference between the theoretical and the real principal stress rotation decreased by shearing process, as shown in Fig. 3.

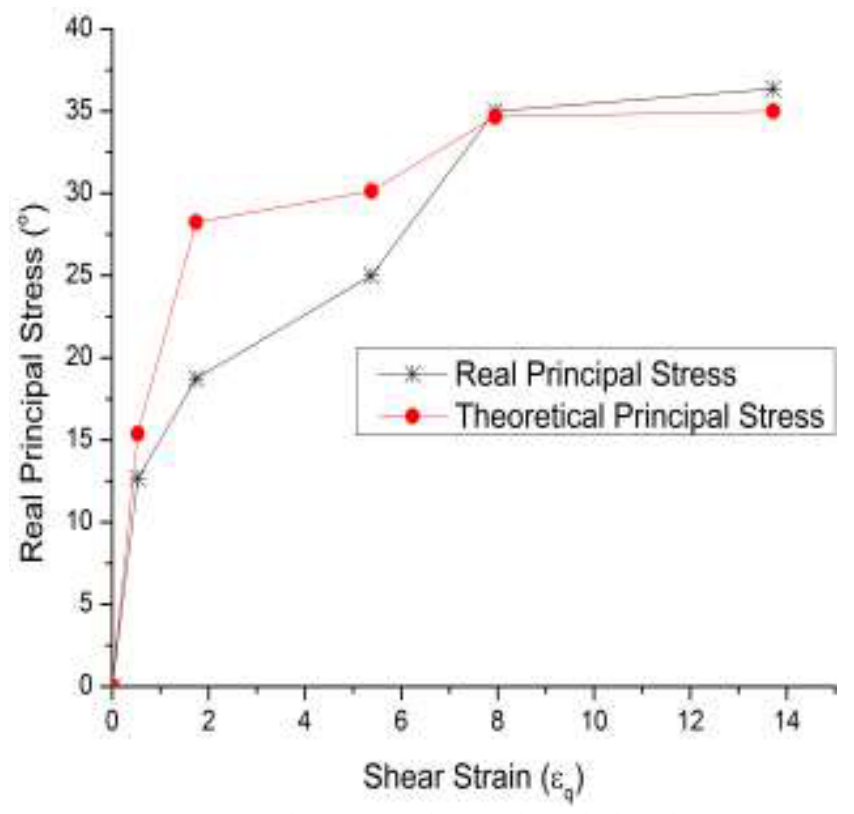

Fig. 3a Difference between the real and the theoretical principal stress rotation for the loose specimen

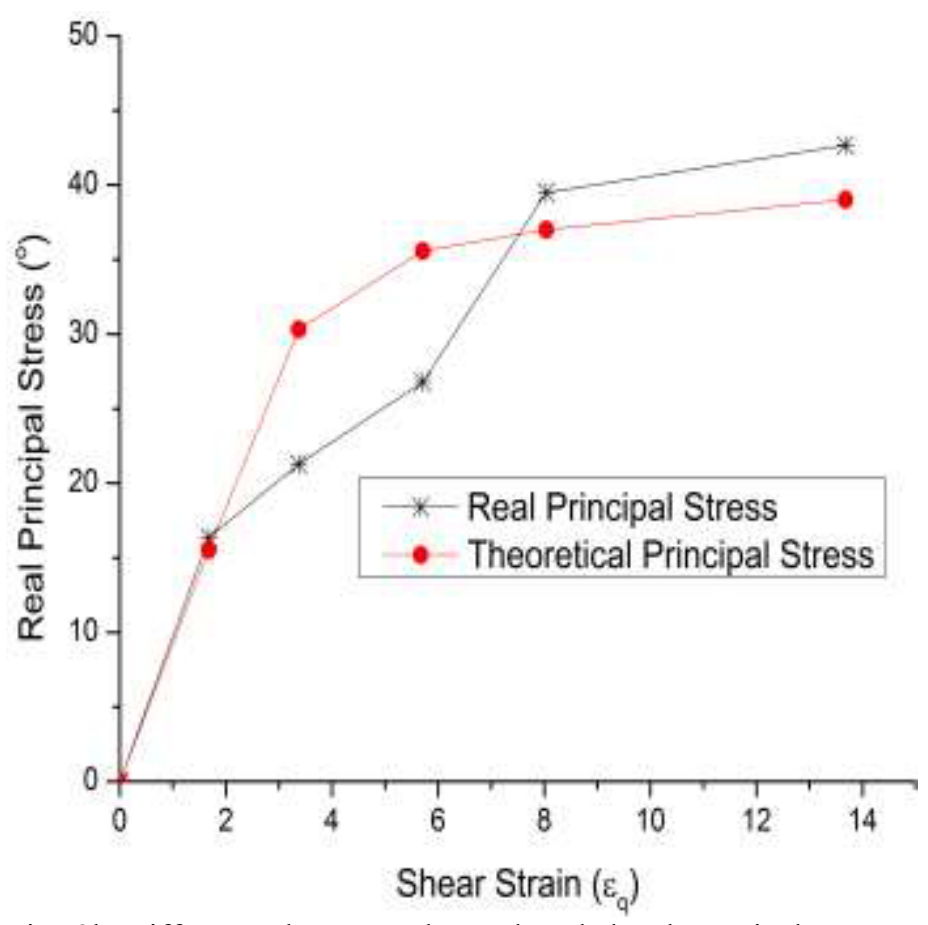

Fig. 3b Difference between the real and the theoretical principal stress rotation for the dense specimen

\section{Conclusion}

Using micromechanical analysis it has been shown that the mobilized stress ratio can be attributed to the interparticle friction angle, $\phi_{\mu}$ and the anisotropic parameters, $\alpha, \beta_{f}$. The Oda $\&$ Konishi's (1974) equation is developed to obtain the direction of the major principal stress by using the macro-level quantities. Micro-level parameters that have been proposed by Oda 
\& Konishi (1974) are not easily obtainable in classical laboratory tests. In this research, however, the suitable micro-level parameters have been chosen to calculate the direction of the major principal stress. The equation was proposed to obtain the real direction of the major principal stress during shear process in the simple shear test. The experimental tests reveal the accuracy of the proposed equations.

\section{References}

1. M. Oda, J. Konishi, Soils \& Found., 14, 3953(1974)

2. M. Oda, Soils \& Found., 15, 17-29 (1975)

3. J.R.F. Arthur, K.S. Chua, T. Dunstan, Geotechnique, 27, 13-30 (1977)

4. K. Miura, S. Miura, S. Toki, Soils \& Found., 26, 3652 (1986)

5. L.T. Yang, X. Li, H.S. Yu, D. Wanatowski, Acta Geotech., (2016)

6. K.H. Roscoe, R.H. Bassett, E.R.L. Cole, Proc. Geotech, Conf., Olso, 231-237 (1967)

7. Wong, J.R.F. Athur, Geotechnique, 36, 215-226 (1986)

8. M. Gutierrez, K. Ishihara, I. Towhata, Soils Found., 31, 121-131 (1991)

9. H. Shavedri, M.R. Taha, F. Kalantary, Gran. Matter, 16, 675-685 (2014)

10. M. Oda, Soils Found., 12, 17-36 (1972)

11. Cai, H.S. Yu, D. Wanatowski, X. Li, J. Geotech. Geoenviron. Eng., 139, 1381-1395 (2013)

12. L. Rothenburg, J. Bathurst, Geotechnique, 39, 601614 (1989)

13. M.R. Taha, H. Shaverdi, Mech. Mater., 69, 173-184 (2013)

14. M.R. Horne, Proc. Roy. A., 286, 79-97 (1956)

15. M. Budhu, Geotech. Geoenviron. Eng., 111, 698$711(1985)$

16. Ochiai, Soils Found., 15, 113-118 (1975) 